\title{
Supporting Information for Sodium-Mediated Low-Temperature Synthesis of Monolayers of Molybdenum Disulfide for Nanoscale Optoelectronic Devices
}

\author{
Syed Hamza Safeer ${ }^{\dagger}$, Marcus V. O. Moutinho ${ }^{\ddagger}$, \\ Arthur.R. J. Barreto ${ }^{\dagger}$, Braulio Soares Archanjo\#, Omar Ginoble \\ Pandoli $₫$, Marco Cremona ${ }^{\dagger}$, Marcelo Eduardo Huguenin Maia \\ da Costa $^{\dagger}$, Fernando Lazaro Freire ${ }^{\dagger}$ and Victor Carozo*,
}

${ }^{\dagger}$ Departamento de Física, Pontifícia Universidade Católica do Rio de Janeiro, Rio de Janeiro, RJ 22451900, Brasil.

‡ Núcleo Multidisciplinar de Pesquisas em Computação NUMPEX-COMP, Campus Duque de Caxias, Universidade Federal do Rio de Janeiro Duque de Caxias, RJ 25240-005, Brasil.

\# Divisao de Metrologia de Materiais, Instituto Nacional de Metrologia, Qualidade e Tecnologia (INMETRO), Duque de Caxias, Rio de Janeiro, RJ 25250020, Brasil.

- Grupo de nanoquímica e química de fluxo, Departamento de Química, Pontifícia Universidade Católica do Rio de Janeiro, Rio de Janeiro, RJ 22451900, Brasil.

*To whom correspondence should be addressed; E-mail: vcarozo@puc-rio.br. 


\section{Single Crystals}
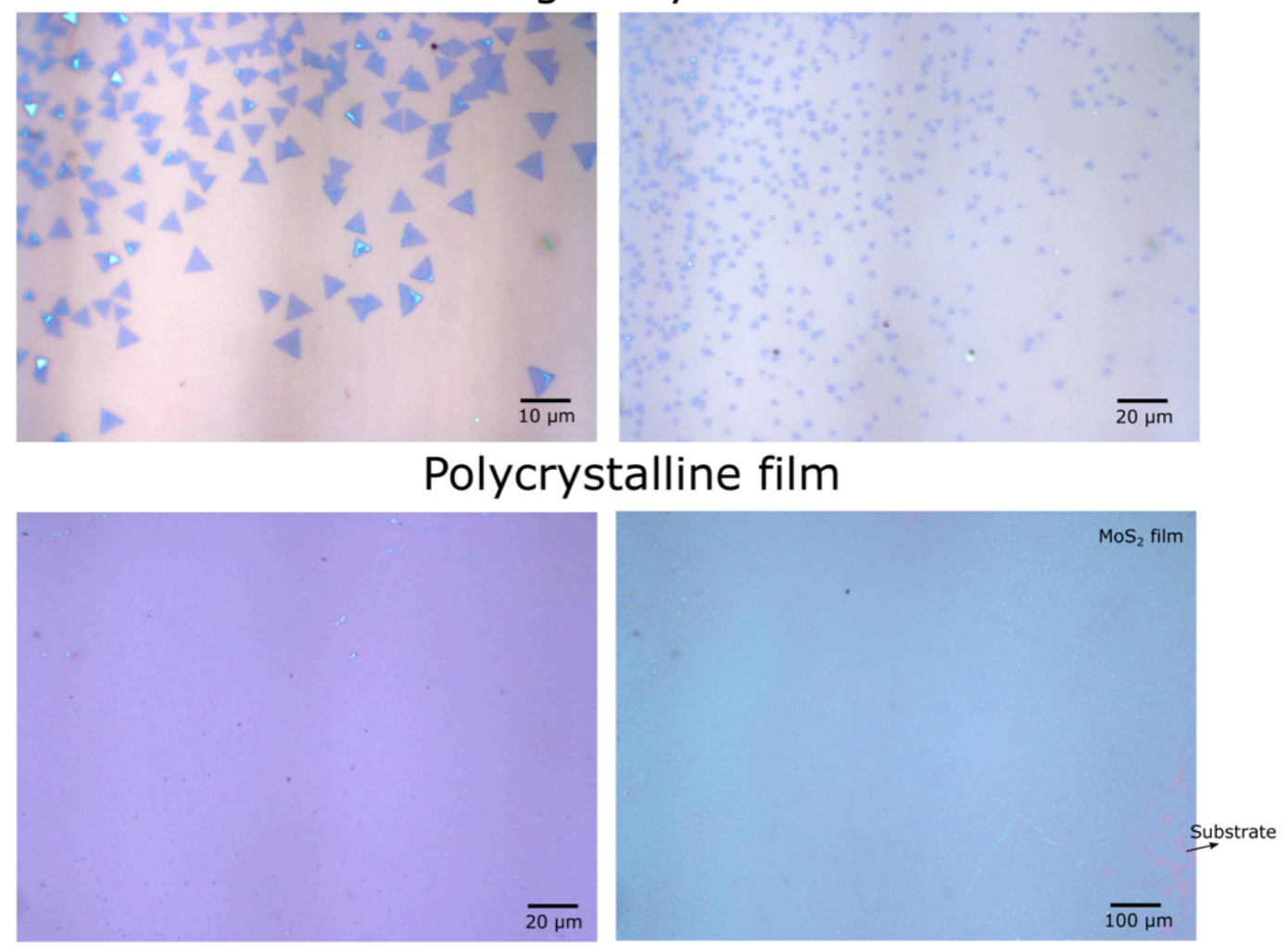

Figure S1. Optical images of monolayers. On top singles crystals of $\mathrm{MoS}_{2}$ and bottom the polycrystalline films. 

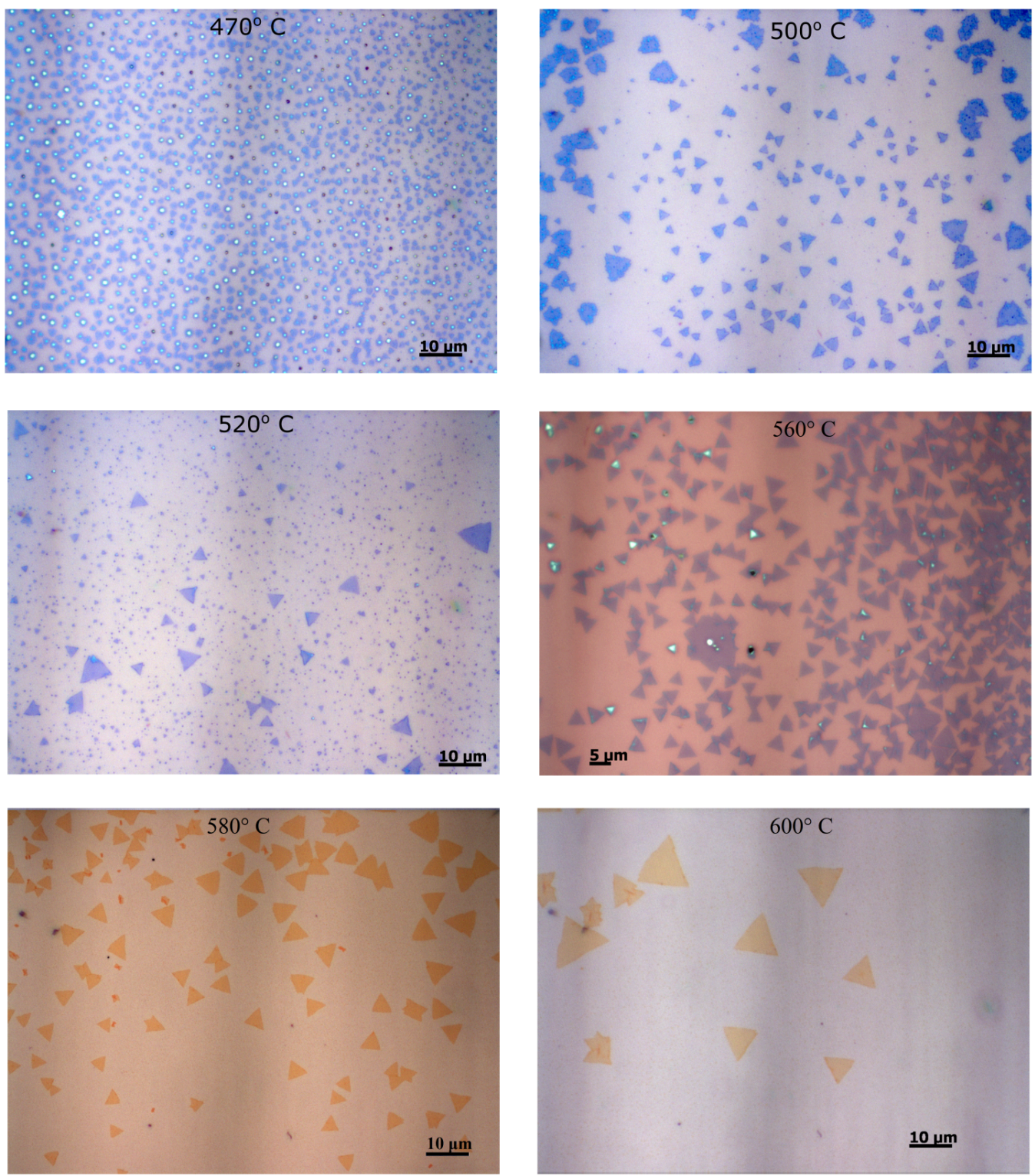

Figure S2. Optical images for six growths temperatures 

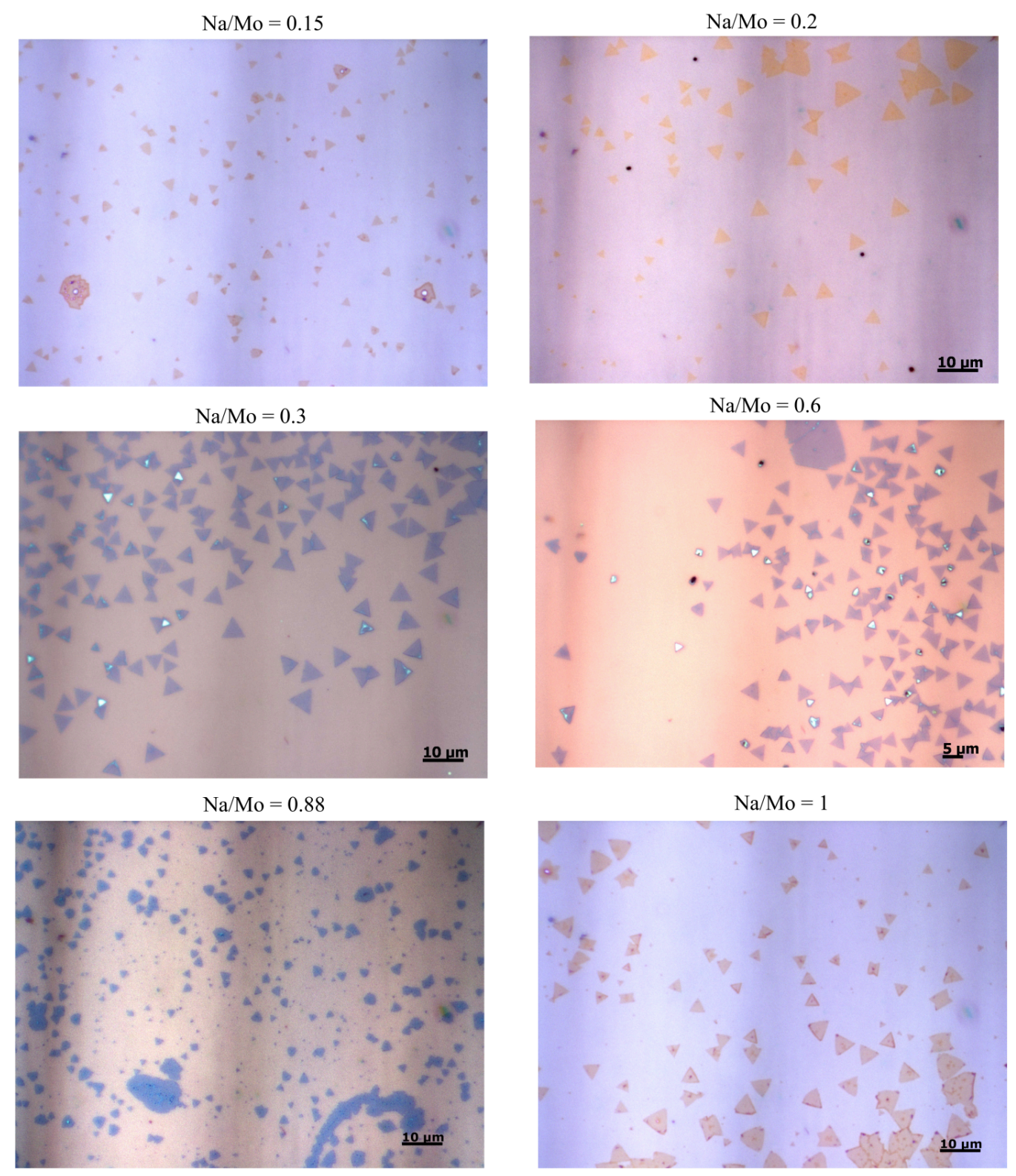

Figure S3(a). Optical images for six Na/Mo precursors' ratio 

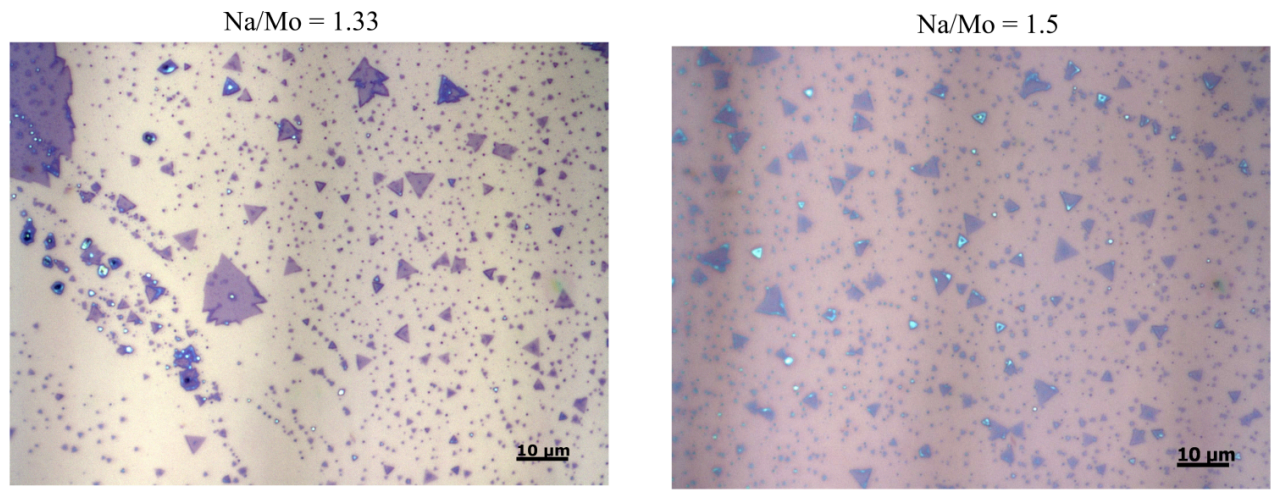

$\mathrm{Na} / \mathrm{Mo}=2$
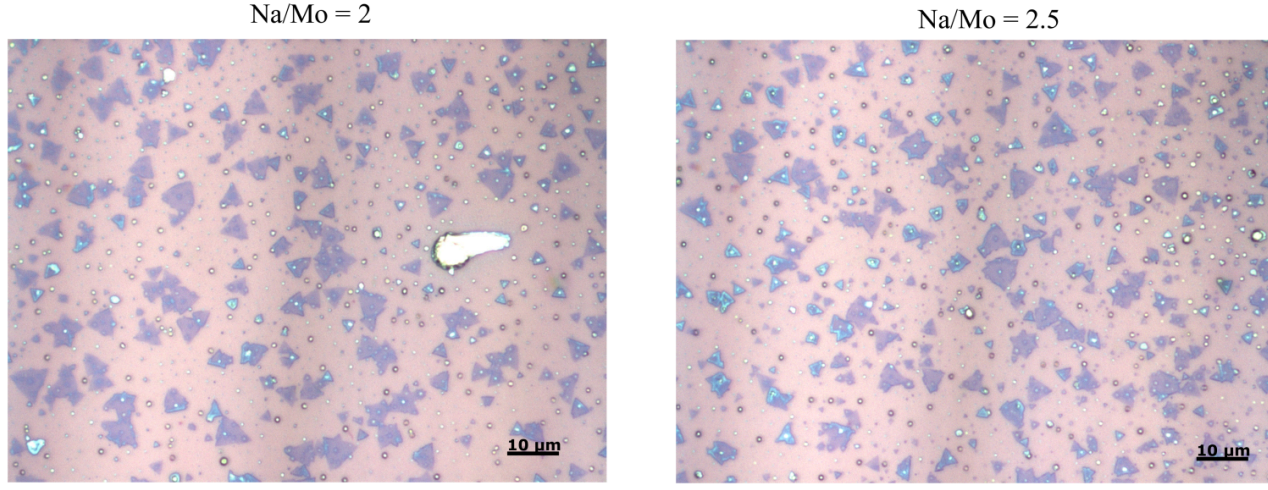

$\mathrm{Na} / \mathrm{Mo}=3$
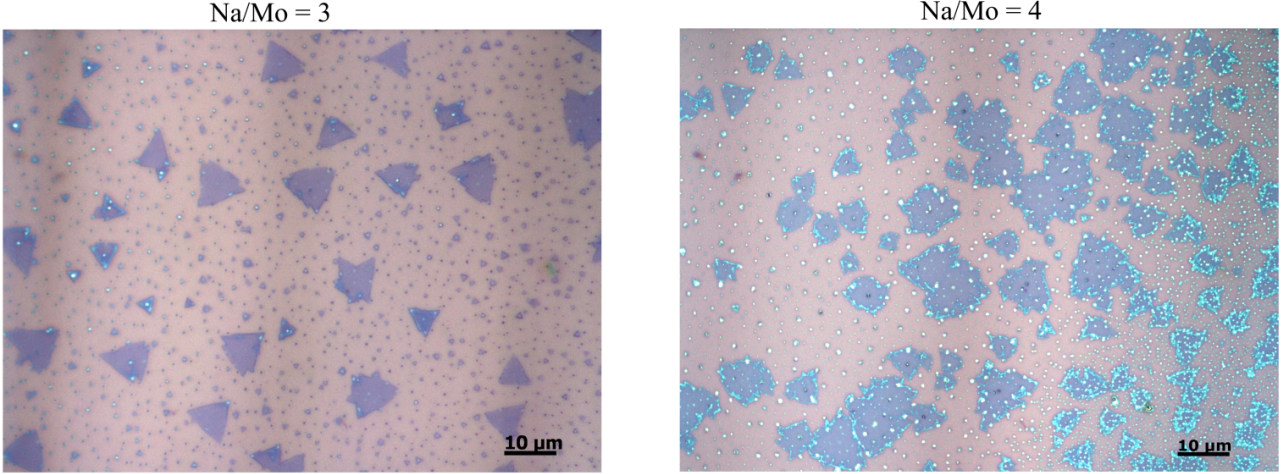

Figure S3(b). Optical images for six Na/Mo precursors' ratio 

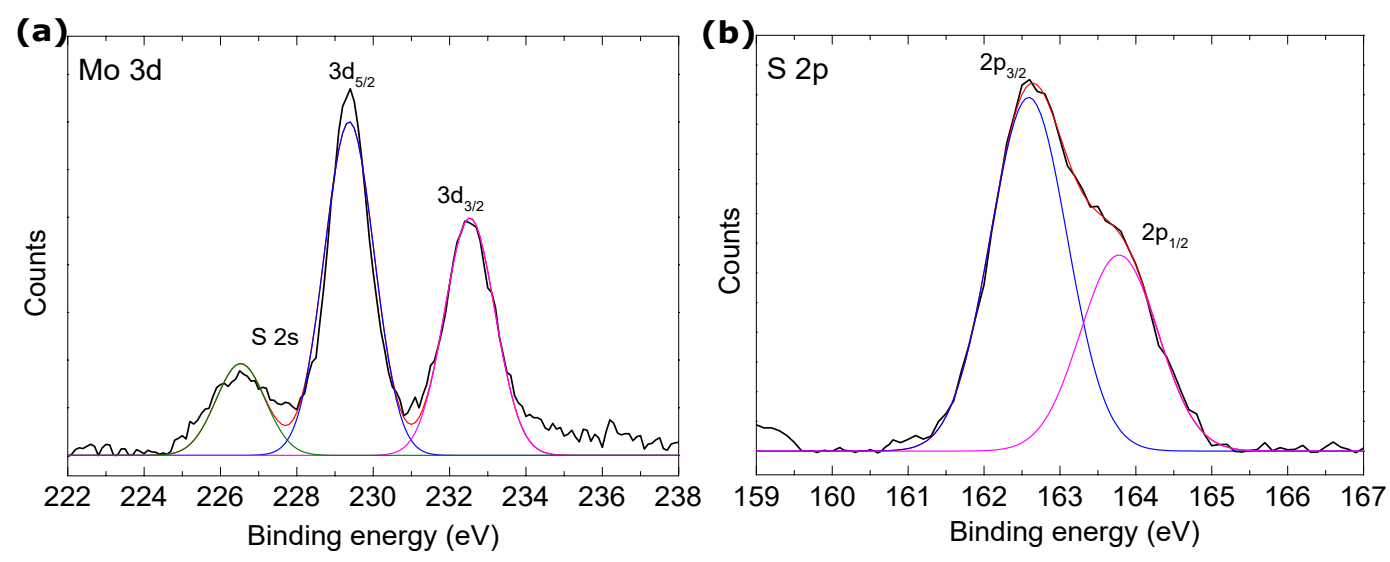

Figure S4. Detailed XPS spectra and fitting of Mo and S peaks. (a) Molybdenum 3d spectrum of $\mathrm{MoS}_{2}$ grown on $\mathrm{SiO}_{2} / \mathrm{Si}$ substrate. (b) $\mathrm{S}$ $2 \mathrm{p}$ spectrum of the same sample. 
(a)

(d) (b)

(e) (c)

(f)

Figure S5. Final structures for large concentrations of $\mathrm{Na}$ in $\mathrm{MoS}_{2}$. (a-c) top view of the $3 \times 3 \mathrm{MoS}_{2}$ supercell with 7,8 and $9 \mathrm{Na}$ atoms, respectively. (d-f) side view of the same structures in (a-c), respectively. The average distance between $\mathrm{Na}$ atoms and the plane with Mo are approximately $4.0 \AA$ (first layer) and $5.8 \AA$ (second layer). For the low concentration regimes (i.e. $<0.45)$ the average distance vary approximately linearly between $3.7 \AA$ and $4.0 \AA$ with the number of the $\mathrm{Na}$ atoms in the supercell. 
(a)

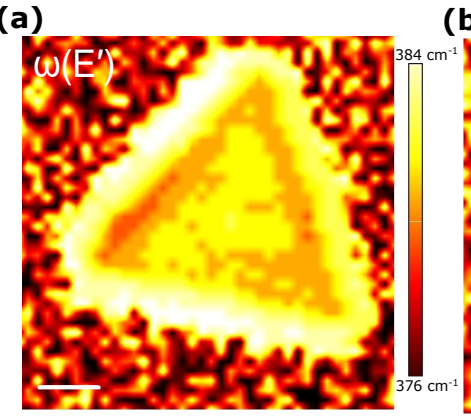

(d)

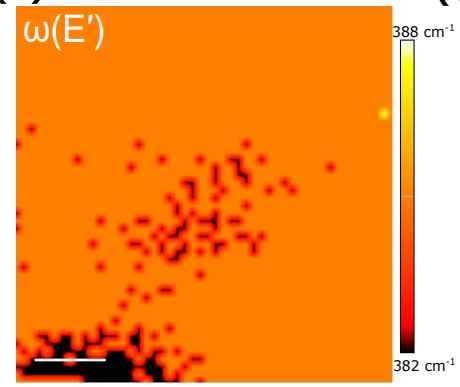

(b)

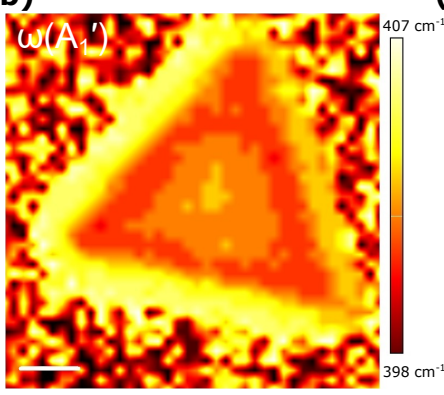

(c)

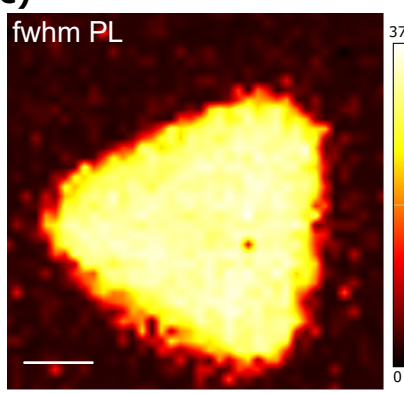

(e)

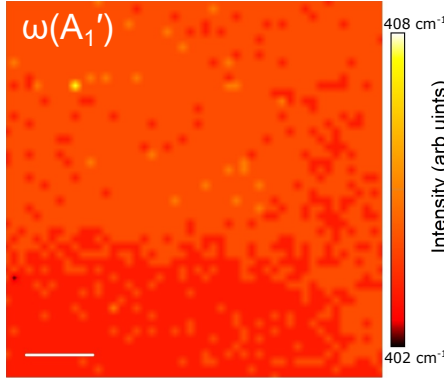

(f)

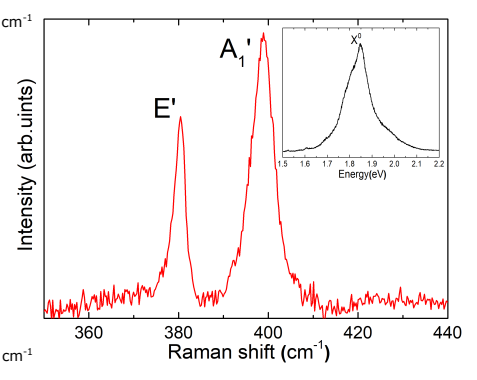

Figure S6. Raman frequency maps of single crystal and polycrystal. (a) The peak position maps of $\mathrm{E}^{\prime}$ and (b) $\mathrm{A}_{1}^{\prime}$ modes of $\mathrm{MoS}_{2}$ grown on $\mathrm{SiO}_{2}$ showing uniformity throughout the triangles area. (c) fwhm of Photoluminescence. (d) The peak position maps of $\mathrm{E}^{\prime}$ and (e) $\mathrm{A}_{1}^{\prime}$ modes of $\mathrm{MoS}_{2}$ polycrystal. (f) Raman spectra of $\mathrm{MoS}_{2}$ polycrystal inset is its photoluminescence. Scale bar $3 \mu$ 
(a)

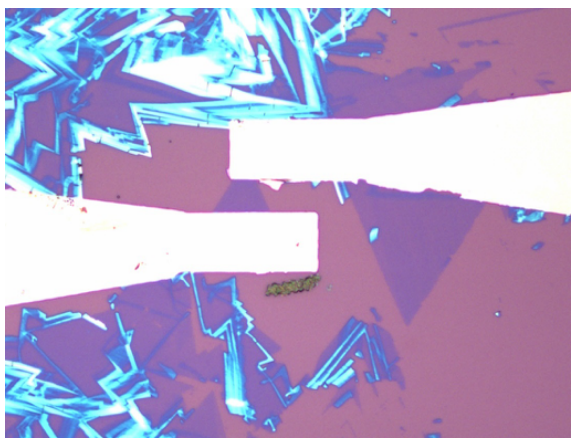

(c)

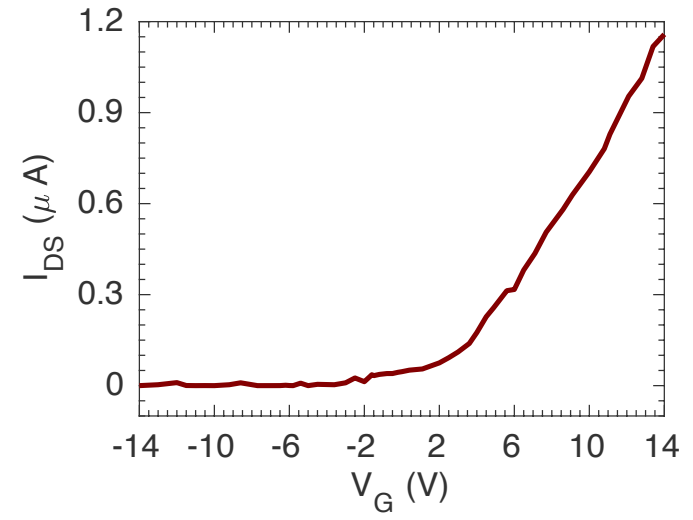

(b)

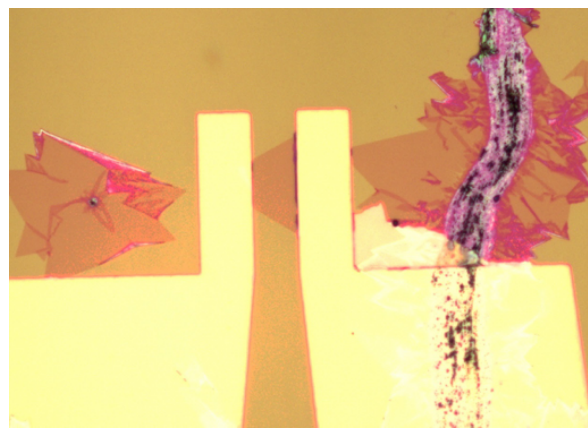

(d)

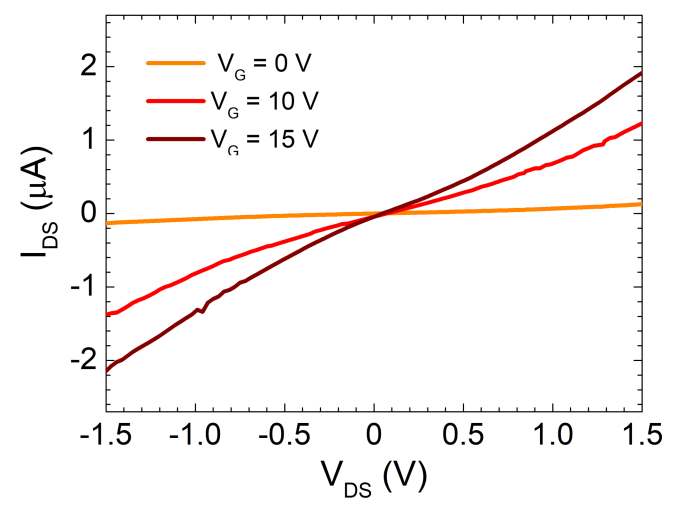

Figure S7. Optical and Transport measurements.(a) and (b) Optical images of two devices. (c) Transfer curve $\left(\mathrm{I}_{D S}\right.$ vs. $\left.\mathrm{V}_{G}\right)$ with $\mathrm{V}_{D S}$ fixed at 1 V. (d) Output curve $\left(\mathrm{I}_{D S}\right.$ vs. $\left.\mathrm{V}_{D S}\right)$ for different $\mathrm{V}_{G}$ values. 

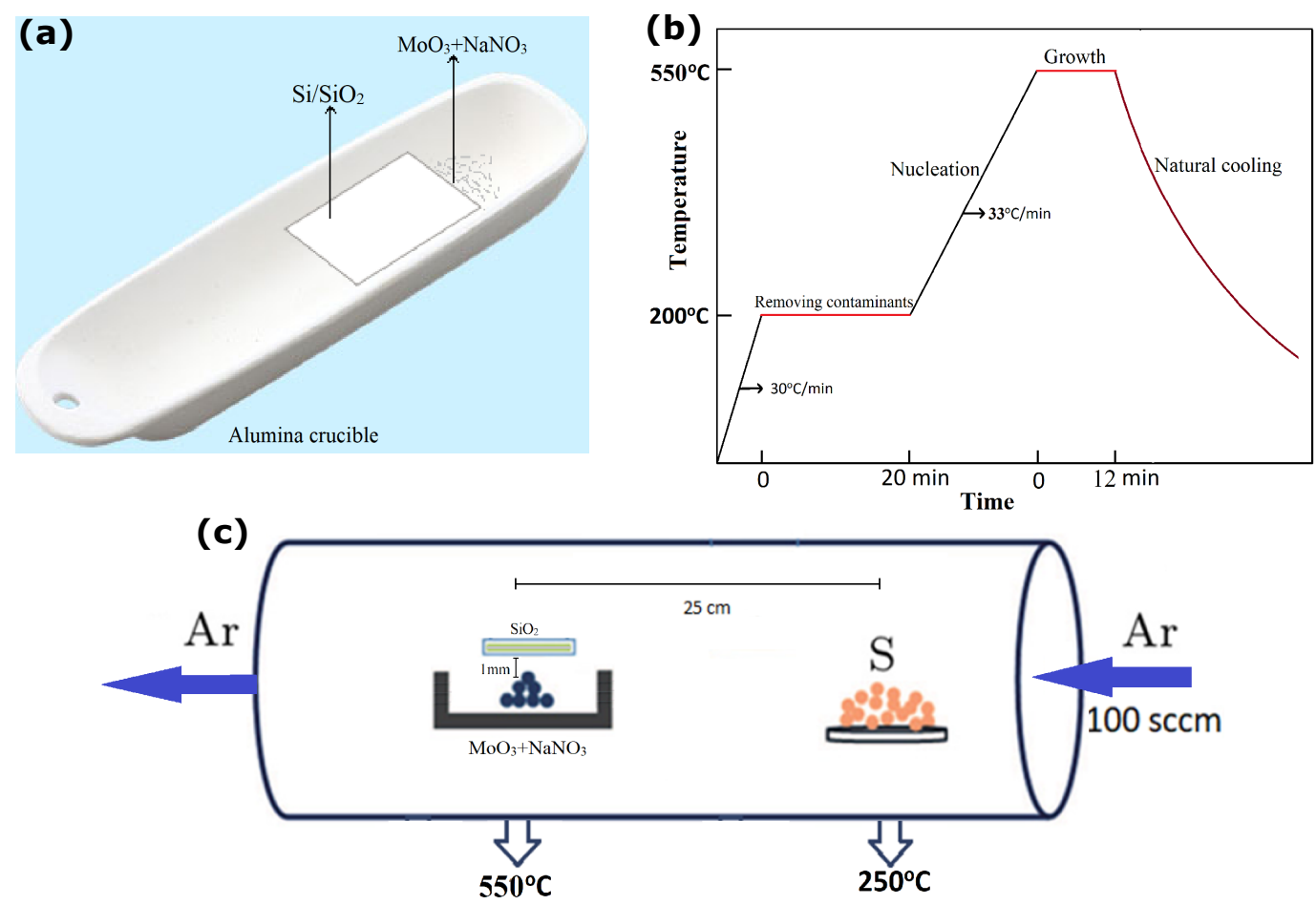

Figure S8. Schematics for the atmospheric pressure CVD synthesis and temperature profile the growth process. (a) Alumina crucible the substrate is placed inside a $\mathrm{mm}$ above, and facing down towards the powder. (b) Temperature profile of whole growth process. (c) Schematics for the APCVD synthesis of $\mathrm{MoS}_{2}$ inside a quartz tube. 